\title{
A differential formulation of thermal constraint release for entangled linear polymers
}

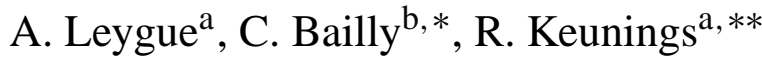 \\ ${ }^{a}$ CESAME, Université Catholique de Louvain, Division of Applied Mechanics, Batiment Euler, B-1348 Louvain-la-Neuve, Belgium \\ ${ }^{\mathrm{b}}$ Unité de Chimie et de Physique des Hauts Polymères, Université Catholique de Louvain, B-1348 Louvain-la-Neuve, Belgium
}

\begin{abstract}
We present a new differential formulation of the thermal constraint release phenomenon for linear entangled polymers. This new formulation predicts a relaxation modulus identical to that predicted by the double reptation theory of Tsenoglou [C. Tsenoglou, Viscoelasticity of binary polymer blends, ACS Polym. Prepr. 28 (1987) 185-186] or Des Cloizeaux [J. Des Cloizeaux, Double reptation vs simple reptation in polymer melts, J. Europhys. Lett. 5 (1988) 437-442] for both monodisperse and polydisperse systems. Additionally, we discuss a simple approximation of our approach as well as its possible use for building simple constitutive equations that account for constraint release in a polydisperse environment.
\end{abstract}

Keywords: Constraint release; Double reptation; Mixing rule; Constitutive equation

\section{Introduction}

Following the introduction on the reptation picture by De Gennes [3], Doi and Edwards [4] proposed a tube-based theory to explain the linear rheology of linear entangled polymers. The numerous variations of the tube model have now reached a high level of maturity and are now capable of a quantitative description of the linear viscoelastic properties of linear entangled polymers (see e.g. [5-9]). The success of these theories is such that the focus is now on the prediction of the linear viscoelastic response of systems with a much more complex architecture, e.g. symmetric [10] or asymmetric stars [11], and mixtures of star and linear polymers $[12,13]$. The key of these successes essentially is an accurate description of: (i) the reptation dynamics (if present), (ii) the fluctuations of the length of the tube [4], and (iii) thermal constraint release phenomena [14], which is a closure to the mean

\footnotetext{
* Corresponding author.

** Co-corresponding author. Tel.: +32 1047 2087; fax: +32 10472180 .

E-mail addresses: bailly@poly.ucl.ac.be (C. Bailly),

roland.keunings@inma.ucl.ac.be (R. Keunings)
}

field approximation of the tube. Accounting for the coupling between those effects is of critical importance. For linear polymers, the distinction made between reptation and contour length fluctuations is somehow arbitrary as these are only different modes of a one-dimensional Rouse chain escaping a tube. This distinction has recently been made thinner by Graham et al. [15] who proposed to model the relaxation through reptation and contour length fluctuations using a modified diffusion process, where diffusivity is position-dependent along the primitive path. Thermal constraint release is today handled efficiently either through dynamic dilution or through double reptation [2,1]. The latter theory can actually be viewed as an approximation of Rouse tube motion induced by constraint release events. In the monodisperse case, Likhtman et al. [8] have showed how double reptation is a good approximation of Rouse tube motion. The mixing rule induced by double reptation has also been recovered within the implementation of constraint release found in a stochastic full chain reptation model developed by Hua et al. [16].

A more complete theory of constraint release involving both tube dilation and rouse tube motion has been proposed by Viovy et al. [17]. 
In the present text, we present a new mathematical formulation of the thermal constraint release phenomenon for monodisperse systems, which we prove to be equivalent to the double reptation theory of Des Cloizeaux [2] and Tsenoglou [1]. This new formulation is then extended to the case of polydisperse systems where it yields a mixing rule identical to the one of double reptation. In parallel, we propose a simple approximation of our theory, which can easily be used as a starting point for building non-linear constitutive equations of the differential type. Finally, we show that our approach to constraint release can simplify integral non-linear integral constitutive equations of polydisperse systems such as the extension proposed by Pattamaprom and Larson [18] of the so-called MLD model of Mead et al. [19].

\section{Classical double reptation}

In this section, we first recall basic elements of reptation theory and its extension to double reptation. In a fixed network of entanglements, reptation theory $[3,4]$ suggests that a polymeric chain is constrained in a tube-like region and therefore can only relax through curvilinear diffusion along its primitive path. Neglecting length fluctuations of the primitive path, one can describe the relaxation of the chain (or equivalently the destruction/renewal of the tube) through the following diffusion equation [4]:

$$
\begin{aligned}
& \frac{\partial P_{0}}{\partial t}=\alpha_{\mathrm{d}} \frac{\partial^{2} P_{0}}{\partial s^{2}}, \\
& P_{0}(t,-1)=0, \\
& P_{0}(t, 1)=0 \text { for } t>0, \\
& P_{0}(0, s)=1 \text { for }-1<s<1,
\end{aligned}
$$

where the equilibrium length of the primitive chain has been normalized to 2 and $\alpha_{\mathrm{d}}$ is a characteristic diffusion constant scaling like the inverse of the cube of the molecular mass. The subscript ' 0 ' indicates that we do not consider any constraint release yet.

The quantity $P_{0}\left(t^{*}, s\right)$ is the probability for a tube segment with curvilinear position $s$ along the primitive chain of not having relaxed between $t=0$ and $t=t^{*}$.

From $P_{0}$, we define the relaxation kernel $K_{0}$ as

$K_{0}(t)=\frac{1}{2} \int_{-1}^{1} P_{0}(t, s) \mathrm{d} s$,

which is but the average of $P_{0}$ over $s$. Using the analytical solution of (1), the relaxation kernel $K_{0}(t)$ can be found to be [4]:

$K_{0}(t)=\frac{8}{\pi^{2}} \sum_{p \text { odd }} \frac{1}{p^{2}} \exp \left(-\frac{p^{2} t}{\tau_{\mathrm{d}}}\right)$,

where the longest relaxation time $\tau_{\mathrm{d}}$ is defined as

$\tau_{\mathrm{d}}=\frac{4}{\pi^{2} \alpha_{\mathrm{d}}}$.
Under simple reptation the relaxation modulus $G_{0}(t)$ is proportional to the relaxation kernel:

$G_{0}(t)=G_{N}^{0} K_{0}(t)$,

where $G_{N}^{0}$ is the plateau modulus.

Introduced by Tsenoglou [1] and Des Cloizeaux [2], double reptation takes into account the mutual interactions of relaxing chains. In a sense, it is a closure to the mean field approximation of the tube. If one assumes that chains interact in a binary fashion (through entanglements), then whenever a chain segment relaxes through reptation, another segment must also relax through thermal constraint release. For monodisperse linear entangled polymers, this simply gives the following relaxation modulus [2]:

$G^{*}(t)=G_{N}^{0}\left(K_{0}(t)\right)^{1+\gamma}$,

where $\gamma$ should be equal to unity, but is often left as an adjustable parameter. A classical choice is to take $\gamma$ slightly above unity $(\gamma=4 / 3)[20]$.

Except for the factor $G_{N}^{0}, G^{*}(t)$ is the power $(1+\gamma)$ of $G_{0}(t)$. As $G_{0}(t)$ is often approximated by its dominant exponential, a crude approximation of double reptation is therefore to simply divide the characteristic relaxation time $\tau_{\mathrm{d}}$ by $(1+\gamma)$.

In a polydisperse system, double reptation provides a mixing rule for predicting the relaxation modulus based on the relaxation kernel of all the present species:

$G^{*}(t)=G_{N}^{0}\left(\sum_{i=1} \phi^{(i)} K_{0}^{(i)}(t)\right)^{1+\gamma}$

where $\phi^{(i)}$ is the volume fraction of species $i$, and $K_{0}^{(i)}$ its relaxation kernel. The disengagement time $\tau_{\mathrm{d}}^{(i)}$ of species $(i)$ is then assumed to be proportional to a particular power of the molecular weight $M^{(i)}$ of the species. This use of double reptation as a mixing rule is quite common and has been proved to give good predictions of the relaxation modulus as a function of the molecular weight distribution of the sample (see e.g. [6,7]).

\section{Constraint release in monodisperse systems}

In this section, we propose a modification to Eq. (1) in order to simultaneously account for reptation and constraint release through double reptation. We will not address the issue of contour length fluctuations in this work as we wish to focus on the thermal constraint release phenomenon only. The incorporation of contour length fluctuations might indeed lead us to modify the typical diffusion operator of the reptation theory.

The classical approach of double reptation is based on the principle of first computing the relaxation kernel of a chain relaxing in a fixed network, and then modifying it in order to take into account the effect of the surrounding relaxing 
chains. This two-step method is not suited for the construction of non-linear constitutive equations, as the mixing rule is non-linear, and does not offer a dynamical description of the processes involved. Instead, we would like to find a differential problem, similar to Eq. (1), such that it would describe the relaxation dynamics of the different segments of the primitive chain under reptation and thermal constraint release.

As any segment of the primitive chain can relax either through reptation or constraint release, we choose to add a second term on the right-hand side of Eq.(1) to account for the latter phenomena. This new term is constructed as follows: Assuming that thermal constraint release can occur with the same probability on any unrelaxed segment [14], it must have an intensity, along the chain, proportional to the fraction of locally unrelaxed segments. This leads to the following differential problem:

$$
\begin{aligned}
& \frac{\partial P_{\gamma}}{\partial t}=\alpha_{\mathrm{d}} \frac{\partial^{2} P_{\gamma}}{\partial s^{2}}+\beta P_{\gamma}, \\
& P_{\gamma}(t,-1)=0, \\
& P_{\gamma}(t, 1)=0 \text { for } t>0, \\
& P_{\gamma}(0, s)=1 \text { for }-1<s<1,
\end{aligned}
$$

where $\beta$ is the rate of thermal constraint release. Following the concept of double reptation, we make this rate proportional to the rate of relaxation through reptation:

$\beta=\gamma \frac{\int_{-1}^{1} \alpha_{\mathrm{d}} \frac{\partial^{2} P_{\gamma}}{\partial s^{2}} \mathrm{~d} s}{\int_{-1}^{1} P_{\gamma} \mathrm{d} s}$,

where the parameter $\gamma$ describes the fact that the relaxation of one chain segment through reptation might induce the relaxation of more than one other segment $(\gamma \geq 1)$. We will prove later that $\gamma$ actually is the same as is Eq. (6).

Another interpretation of Eqs. (8) and (9) is the following: For any tube segment that relaxes on a given chain through reptation, there is another tube segment that will relax. While the first disappearing segment will be located at a chain end, the second has to be picked randomly among all the unrelaxed tube segments. The expression $\int_{-1}^{1} \alpha_{\mathrm{d}} \frac{\partial^{2} P_{\gamma}}{\partial s^{2}} \mathrm{~d} s$ simply measures the rate of relaxation through reptation, while the function $\frac{P_{\gamma}}{\int_{-1}^{1} P_{\gamma} \mathrm{d} s}$ describes how this rate of relaxation should be redistributed along the chain; i.e. proportionally to the local fraction of unrelaxed chain segments (or surviving tube segments).

It can be verified by simple substitution that Eqs. (8) and (9) have the following analytical solution:

$$
\begin{aligned}
P_{\gamma}(t, s) & =P_{0}(t, s)\left(\frac{1}{2} \int_{-1}^{1} P_{0}\left(t, s^{\prime}\right) \mathrm{d} s^{\prime}\right)^{\gamma} \\
& =P_{0}(t, s)\left(K_{0}(t)\right)^{\gamma}
\end{aligned}
$$

which is a product of two factors where the first one can be interpreted as accounting for the relaxation of a chain in a fixed environment, while the second one would account for the relaxation of the environment.
Finally, we define the relaxation modulus $G_{\gamma}(t)$ as proportional to the fraction of unrelaxed chain segments:

$G_{\gamma}(t)=G_{N}^{0} \frac{1}{2} \int_{-1}^{1} P_{\gamma}(t, s) \mathrm{d} s$.

This approach to constraint release predicts exactly the same relaxation modulus as double reptation. The equivalence of both formulations is easily seen through the comparison of Eqs. (6) and (11), where $P_{\gamma}$ has been substituted by its analytical expression (10). We also see that the parameter $\gamma$ has the same meaning in both formulas and that the expression (9) for $\beta$ can be simplified as

$\beta(t)=\gamma \frac{\frac{\partial K_{0}(t)}{\partial t}}{K_{0}(t)}$

In describing the relaxation of a polymeric system one might not only be interested in the relaxation modulus $G_{\gamma}(t)$ but also in the dynamics of relaxation through reptation and constraint release along the chain. Such information is needed, for example, in the full contour-variable reptation model proposed by Mead et al. [19]. Under simple reptation, tube segments are renewed at the chain's ends only, while thermal constraint release allows the renewal of the orientation of internal tube segments. If one neglects constraint release $(\gamma=0)$, the variation of $P_{0}(t, s)$ is only due to a flux from the inside of the chain towards the chain's ends, where all the actual relaxation occurs. When $\gamma$ is not zero, $P_{\gamma}(t, s)$ also decreases due to a local relaxation, and the total loss along the chain is $\gamma$ times the loss at the chain's ends. This difference in the dynamics of internal segments can be best observed at early times when looking at the relaxation of the segments at the center of the chain. In Fig. 1, we compare $P_{0}(t, s)$ and $P_{\gamma}(t, s)$ at the center of the chain; i.e. $s=0$ and for $\gamma=1$. At early times, $P_{0}(t, 0)$ exhibits a plateau which comes from the fact that inner segments can only relax through reptation and must therefore wait for the chain to

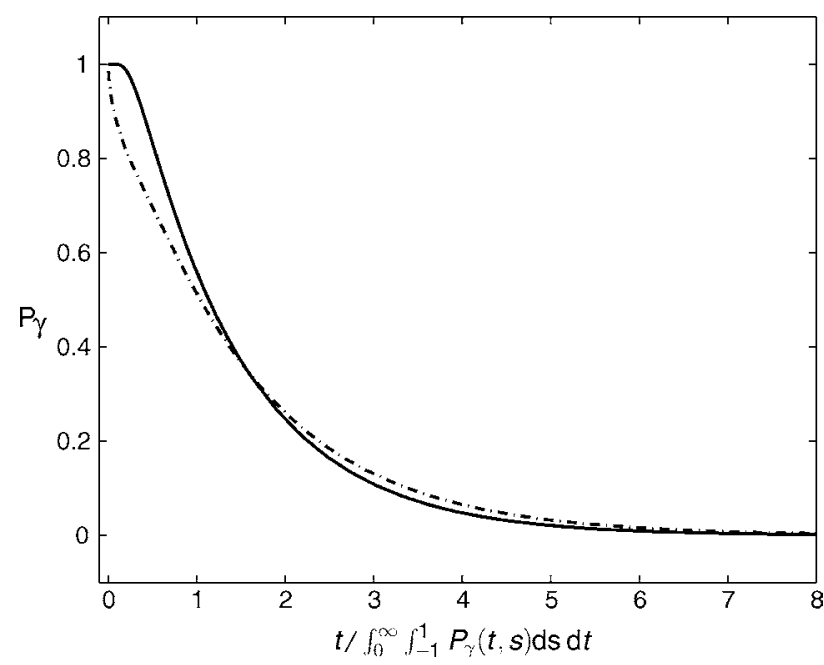

Fig. 1. Comparison of the relaxation dynamics at the center of the primitive chain with $\left(P_{1}(t, 0): \cdots-\cdot\right)$ and without $\left(P_{0}(t, 0):-\right)$ constraint release. 
reptate far enough before having the opportunity to relax. On the other hand, we see that $P_{\gamma}(t, 0)$ does not have that feature, as some inner segments can relax through thermal constraint release as soon as the outer segments relax through reptation.

\section{Construction of a simple linear viscoelastic model}

From the partial differential equation (PDE) (8) we proposed for the evolution of $P_{\gamma}$, it is possible to build a linear viscoelastic model that accounts for both reptation and constraint release in simple shear flows. Let us define $\sigma(t, s)$ as the non-dimensional stress carried at time $t$ by tube segments at position $s$ along the primitive path.

$\sigma(t, s)=\int_{-\infty}^{t} P_{\gamma}\left(t-t^{\prime}, s\right) \dot{\gamma}_{x y}\left(t^{\prime}\right) \mathrm{d} t^{\prime}$,

where $\dot{\gamma}_{x y}\left(t^{\prime}\right)$ is the shear rate at time $t^{\prime}$. This simple linear model reads:

$$
\begin{aligned}
& \frac{\partial \sigma}{\partial t}=\dot{\gamma}+\alpha_{\mathrm{d}} \frac{\partial^{2} \sigma}{\partial s^{2}}+\int_{-\infty}^{t} \beta\left(t-t^{\prime}\right) P_{\gamma}\left(t-t^{\prime}, s\right) \dot{\gamma}_{x y}\left(t^{\prime}\right) \mathrm{d} t^{\prime} \\
& \sigma(t,-1)=0 \\
& \sigma(t, 1)=0 \text { for } t>0 \\
& \sigma(0, s)=0 \text { for }-1<s<1 \\
& \beta(t)=\gamma \frac{\frac{\partial K_{0}(t)}{\partial t}}{K_{0}(t)} \\
& \tau_{x y}(t)=G_{N}^{0} \frac{1}{2} \int_{-1}^{1} \sigma(t, s) \mathrm{d} s
\end{aligned}
$$

where $\tau_{x y}$ is the shear stress.

In order to obtain from (14) and (15) a simple differential linear model we approximate the relaxation kernel $K_{0}(t)$ appearing in Eq. (15) by a single decreasing exponential with characteristic time $\gamma \tau_{\mathrm{cr}}$. The new model simply reads:

$$
\begin{aligned}
& \frac{\partial \sigma}{\partial t}=\dot{\gamma}+\alpha_{\mathrm{d}} \frac{\partial^{2} \sigma}{\partial s^{2}}-\frac{\sigma}{\tau_{\mathrm{cr}}}, \\
& \sigma(t,-1)=0, \\
& \sigma(t, 1)=0 \text { for } t>0, \\
& \sigma(0, s)=0 \text { for }-1<s<1, \\
& \tau_{x y}=G_{N}^{0} \frac{1}{2} \int_{-1}^{1} \sigma \mathrm{d} s .
\end{aligned}
$$

The response of this simplified model after a unit step strain is

$\sigma(t, s)=P_{0}(t, s) \exp \left(-\frac{t}{\tau_{\mathrm{cr}}}\right)$.

The scalar $\tau_{\mathrm{cr}}$ is of the order of $\tau_{\mathrm{d}}$ and represents the average disentanglement time through thermal constraint release phenomena. Its value is chosen such that Eqs. (14) and (16) yield the same zero-shear viscosity. From the analytical solution of (16), we find that $\tau_{\mathrm{cr}}$ is the solution of the

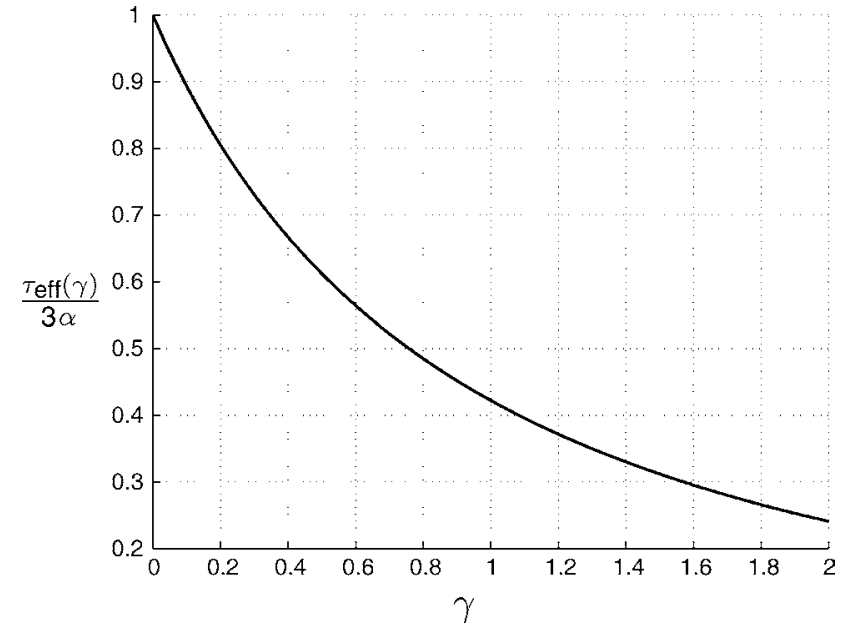

Fig. 2. Normalized effective relaxation time as a function of $\gamma$.

following equation:

$$
\int_{0}^{+\infty}\left(K_{0}(t)\right)^{(1+\gamma)} \mathrm{d} t=\int_{0}^{+\infty}\left(K_{0}(t) \exp \left(-\frac{t}{\tau_{\mathrm{cr}}}\right)\right) \mathrm{d} t .
$$

The approximate model (16) is of little use by itself, but it is a first step towards a more realistic non-linear differential constitutive equation which would have the same structure in terms of differential operators along the $s$ coordinate.

The zero-shear viscosity $\eta_{0}$ predicted by these models is a function of $\alpha_{\mathrm{d}}$ and $\gamma$. It can easily be proved that $\eta_{0}$ scales like $\alpha_{\mathrm{d}}^{-1}$, but does not scale exactly like $(1+\gamma)^{-1}$. For $\gamma=0$ we can prove that $\eta_{0}=\frac{G_{N}^{0}}{3 \alpha_{\mathrm{d}}}$, but for higher values of $\gamma$ we have to compute $\eta_{0}$ numerically. Defining the effective relaxation time $\tau_{\text {eff }}$ as $\tau_{\text {eff }}=\frac{\eta_{0}}{G_{N}^{0}}$, we illustrate in Fig. (2) how this quantity changes with respect to $\gamma$. When $\gamma=1$, the effective relaxation time is less than half $(\approx 0.42)$ the relaxation time when double reptation is ignored. Thermal constraint release is traditionally accounted for in constitutive equations simply by dividing the relaxation time by 2 , which is apparently not enough when one considers the full spectrum of relaxation times instead of the dominant one only.

\section{Constraint release in polydisperse systems}

Using the same approach, the above formulation of constraint release can easily be extended to polydisperse systems.

In the absence of thermal constraint release, we can consider that all species reptate independently. From a modeling point of view, this would lead to a set of uncoupled partial differential equations (PDEs), where each PDE describes a particular species. As soon as constraint release comes into play, the relaxation of the species is coupled because they are each other's environment (including themselves). The key 
issue is therefore to compute consistently the rate $\beta$ of relaxation of the environment.

Assuming that the polydisperse system is made of molecules with a discrete distribution of $N$ masses, let us define $P_{\gamma}^{(i)}(t, s)$ as the tube survival probability for molecules of mass $M^{(i)}$. The time evolution of $P_{\gamma}^{(i)}(t, s)$ is described by the following PDE:

$$
\begin{aligned}
& \frac{\partial P_{\gamma}^{(i)}}{\partial t}=\alpha_{\mathrm{d}}^{(i)} \frac{\partial^{2} P_{\gamma}^{(i)}}{\partial s^{2}}+\beta P_{\gamma}^{(i)}, \\
& P_{\gamma}^{(i)}(t,-1)=0, \\
& P_{\gamma}^{(i)}(t, 1)=0 \text { for } t>0, \\
& P_{\gamma}^{(i)}(0, s)=1 \text { for }-1<s<1 .
\end{aligned}
$$

In view of Eq. (9), the rate of constraint release $\beta$ is consistently defined as

$\beta=\gamma \frac{\sum_{i} \phi^{(i)} \int_{-1}^{1} \alpha_{\mathrm{d}}^{(i)} \frac{\partial^{2} P_{\gamma}^{(i)}}{\partial s^{2}} \mathrm{~d} s}{\sum_{i} \phi^{(i)} \int_{-1}^{1} P_{\gamma}^{(i)} \mathrm{d} s}$,

where $\phi^{(i)}$ is the volume fraction of species $(i)$ and $\gamma$ keeps the same meaning as in the monodisperse case. The diffusion coefficient $\alpha_{\mathrm{d}}^{(i)}$ has the form:

$\alpha_{\mathrm{d}}^{(i)}=\frac{4}{\pi^{2} K_{\mathrm{d}}\left(M^{(i)}\right)^{3}}$,

where $K_{\mathrm{d}}$ is an adjustable material parameter and the exponent 3 accounts for the scaling of the disengagement time $\tau_{\mathrm{d}}$ with respect to the molecular mass.

When $\gamma=0$, all PDEs (19)are decoupled and their solutions $P_{0}^{(i)}(t, s)$ can be computed easily as each equation describes the relaxation through simple reptation of a particular species. When $\gamma \neq 0$, Eq.(19) has the following solution:

$P_{\gamma}^{(i)}(t, s)=P_{0}^{(i)}(t, s)\left(\sum_{j} \frac{1}{2} \int_{-1}^{1} \phi^{(j)} P_{0}^{(j)}\left(t, s^{\prime}\right) \mathrm{d} s^{\prime}\right)^{\gamma}$,

and $P_{0}^{(i)}(t, s)$ is the tube survival probability under simple reptation. In the previous expression, the first term accounts for the relaxation of the chains through reptation only, while the second accounts for the relaxation of the environment. Finally, we define the relaxation modulus as

$G_{\gamma}(t)=G_{N}^{0} \sum_{i} \frac{\phi^{(i)}}{2} \int_{-1}^{1} P_{\gamma}^{(i)}(t, s) \mathrm{d} s$.

It is straightforward to check that the resulting modulus is identical to the modulus one would obtain with the mixing rule (7) of double reptation.

Similarly to what we did for monodisperse systems, it is possible to build upon this approach a linear viscoelastic model for polydisperse systems. Defining $\sigma^{(i)}(t, s)$ as the stress carried by chain segments of species $(i)$ and coordinate $s$ along the primitive path, we obtain the following model:

$\frac{\partial \sigma^{(i)}}{\partial t}=\dot{\gamma}+\alpha_{\mathrm{d}} \frac{\partial^{2} \sigma^{(i)}}{\partial s^{2}}+\int_{-\infty}^{t} \beta\left(t-t^{\prime}\right) P_{\gamma}^{(i)}\left(t-t^{\prime}, s\right) \dot{\gamma}_{x y}\left(t^{\prime}\right) \mathrm{d} t^{\prime}$,

$\sigma^{(i)}(t,-1)=0$,

$\sigma^{(i)}(t, 1)=0$ for $t>0$,

$\sigma^{(i)}(0, s)=0$ for $-1<s<1$,

$\beta(t)=\gamma \frac{\sum_{i} \phi^{(i)} \frac{\partial K_{0}^{(i)}(t)}{\partial t}}{\sum_{i} \phi^{(i)} K_{0}^{(i)}(t)}$,

$\tau_{x y}(t)=G_{N}^{0} \sum_{i} \frac{\phi^{(i)}}{2} \int_{-1}^{1} \sigma^{(i)}(t, s) \mathrm{d} s$.

This linear viscoelastic model only needs $N$ objects, namely the $N$ functions $\sigma^{(i)}$ to reproduce the full complexity of thermal constraint release within a polydisperse system of $N$ masses. It is also capable of accounting for an arbitrary mixing exponent $\gamma$. This new formulation of constraint release could therefore be used as a starting point for the implementation of complex integral constitutive equations for polydisperse systems.

Such a constitutive equation has been proposed by Pattamaprom and Larson [18], as an extension of the so-called toy-MLD model by Mead et al. [19]. The implementation of thermal constraint release (or reptative constraint release, following the authors) within the polydisperse version of the toy-MLD model relied on a popular approach in which one accounts for any possible interaction between all $N$ polymeric masses. A first drawback of this method is that it cannot handle cases where $\gamma$ is not equal to unity. Furthermore $N^{2}$ modes (all $M^{(i)} M^{(j)}$ pairs) are required to fully model double reptation. This higher algorithmic complexity can dramatically increase the computational simulation cost when dealing with polymeric systems composed of many different masses. Both these constraints are alleviated when using Eqs. (24)-(26) to implement thermal constraint release.

\section{Conclusions}

We have proposed a new differential formulation of thermal constraint release in monodisperse and polydisperse entangled polymeric systems. The core of our approach is a differential problem that allows the direct computation of the tube survival probability when both reptation and thermal constraint release are active. Although this theory predicts a relaxation modulus mathematically equivalent to the one predicted by the double reptation theory of Tsenoglou [1] and Des Cloizeaux [2], it does not use a non-linear mixing rule, and thus is easier to extend to a full constitutive equation. Progress along this line is the subject of a forthcoming publication. 


\section{Acknowledgments}

This work is supported by the "Action de recherche concertée" programme of the Communauté Française de Belgique and by BASF Aktiengesellschaft.

We wish to thank Giuseppe Marrucci and Evelyne van Ruymbeke for fruitful discussions.

\section{References}

[1] C. Tsenoglou, Viscoelasticity of binary polymer blends, ACS Polym. Prepr. 28 (1987) 185-186.

[2] J. Des Cloizeaux, Double reptation vs simple reptation in polymer melts, J. Europhys. Lett. 5 (1988) 437-442.

[3] P.G. De Gennes, Reptation of a polymer chain in the presence of fixed obstacles, J. Chem. Phys. 55 (1971) 572-579.

[4] M. Doi, S.F. Edwards, The Theory of Polymer Dynamics, Oxford University Press, 1986.

[5] C. Pattamaprom, R.G. Larson, T.J. Van Dyke, Quantitative predictions of linear viscoelastic rheological properties of entangled polymers, Rheol. Acta 39 (6) (2000) 517-531.

[6] F. Léonardi, J.-C. Majesté, A. Allal, G. Marin, Rheological models based on the double reptation mixing rule: the effects of a polydisperse environment, J. Rheol. 44 (2000) 675-692.

[7] E. van Ruymbeke, R. Keunings, V. Stéphenne, A. Hagenaars, C. Bailly, Evaluation of reptation models for predicting the linear viscoelastic properties of entangled linear polymers, Macromolecules 35 (7) (2002) 2689-2699.

[8] A.E. Likhtman, T.C.B. McLeish, Quantative theory for linear dynamics of linear entangled polymers, Macromolecules 35 (2002) 6332-6343.
[9] S.T. Milner, T.C.B. McLeish, Reptation and contour-length fluctuations in melts of linear polymers, Phys. Rev. Lett. 81 (3) (1998) 725-728.

[10] S.T. Milner, T.C.B. McLeish, Parameter-free theory for stress relaxation in star polymer melts, Macromolecules 30 (1997) 2159-2166.

[11] A.L. Frischnecht, S.T. Milner, A. Pryke, R.N. Young, R. Hawkins, T.C.B. McLeish, Rheology of three-arm asymmetric star polymer melts, Macromolecules 35 (2002) 4801-4820.

[12] S.T. Milner, T.C.B. McLeish, R.N. Young, A. Hakiki, J.M. Johnson, Dynamic dilution, constraint-release, and star-linear blends, Macromolecules 31 (1998) 9345-9353.

[13] S.J. Park, R.G. Larson, Tube dilution and reptation in binary blends of monodisperse linear polymers, Macromolecules 37 (2004) 597-604.

[14] G. Marrucci, Relaxation by reptation and tube enlargement: a model for polydisperse polymers, J. Polym. Sci., Polym. Phys. 23 (1985) 159177.

[15] R.S. Graham, A.E. Likhtman, T.C.B. McLeish, S.T. Milner, Microscopic theory of linear entangled polymer chains under rapid deformation including chain stretch and convective constraint release, J. Rheol. 47 (5) 1171-1200 (2003).

[16] C.C. Hua, J.D. Schieber, D.C. Venerus, Segment connectivity, chainlength breathing, segmental stretch, and constraint release in reptation models. I. Theory and single-step strain predictions, J. Chem. Phys. 109 (22) (1998) 10018-10032.

[17] J.L. Viovy, M. Rubinstein, R.H. Colby, Constraint release in polymer melts: tube reorganization versus tube dilation, Macromolecules 24 (12) (1991) 3587-3596.

[18] C. Pattamaprom, R.G. Larson, Constraint release effects in monodisperse and bidisperse polystyrenes in fast transient shearing flows, Macromolecules 34 (2001) 5229-5237.

[19] D.W. Mead, R.G. Larson, M. Doi, A molecular theory for fast flows of entangled polymers, Macromolecules 31 (22) (1998) 7895-7914.

[20] R.H. Colby, M. Rubinstein, Two-parameter scaling for polymers in $\theta$ solvents, Macromolecules 23 (1990) 2753-2757. 